\title{
DESIGN AND CONSTRUCTION OF A WDM TRANSCEIVER WITH VHGT USING HYBRID INTEGRATION TECHNOLOGY
}

\author{
Vítězslav Jeřábek, Václav Prajzler, Karel Bušek, J.A.Arciniega, David Mareš \\ Department of Microelectronics, Faculty of Electrical Engineering, CTU in Prague, \\ Technická 2, Prague 6 \\ E-mail : jerabek@fel.cvut.cz
}

\begin{abstract}
:
We report about design and construction of WDM bidirectional transceiver module (TRx) for the passive optical network (PON) of a fiber to the home FTTH topology. The TRx uses a microoptics hybrid integration technology with volume holographic Bragg grating triplex filter (VHGT) and a collimation lenses for wavelength multiplexing/ demultiplexing. Our optical WDM transceiver TRx has been constructed using system of a four micromodules in the new circle topology. The optical micromodul with VHGT filter, two optoelectronic receivers' micromodules for receiving download information (internet and digital TV signals) and optoelectronic transmitter micromodul for transmitting of a upload information.
\end{abstract}

\section{INTRODUCTION}

The microoptical lightwave circuit (MLC) and planar lightwave circuit (PLC) hybrid integration technology enables us to construct component by combining MLC or PLC with passive function (fiber, planar waveguides, collimation lenses, optical gratings atc.) and active optoelectronic devices (laser diodes, semiconductor optical amplifiers, photodiodes atc.) hybridized on a MLC or PLC [1], [2]. WDM type triplex transceiver TRx is useful for a subscriber part of the passive optical network PON for a fiber to the home FTTH topology. An optical TRx transmits a $1310 \mathrm{~nm}$ radiation upload and receives a $1490 \mathrm{~nm}$ download internet data as well as a $1550 \mathrm{~nm}$ download digital video signals for wavelength division multiplexing WDM cable TV application. The TRx uses a microoptics or planar lightwave hybrid integration technology. For wavelength multiplexing/ demultiplexing (MUX/DEMUX) were used TFF (thin-film filters), optical mirrors and prisms [3].

Our optical WDM transceiver TRx has been constructed using system of a four micromodules in the new circle topology. The triplex optical MUX/DEMUX micromodule was composed of volume holographic Bragg grating triplex filter (VHGT) from Ondax Ltd. This transmission type grating filter has high diffraction efficiency and very low insertion loses and optical crosstalk [4]. The optoelectronic part of TRx is crate of a two microwave OE (optoelectronics) receiver micromodules with bandwidth of $2.5 \mathrm{GHz}$ with design after [5]. The $\mathrm{OE}$ transmitter micromodule uses Fabry-Perot laser diode with a injection microwave modulator and optical power feed back control electronics.

\section{DESIGN AND MEASURE RESULTS OF THE MICROMODULES}

\section{The triplex optical micromodule}

The triplex optical MUX/DEMUX micromodule was composed of VHGT filter made by BK7 glass made by Ondax Ltd. with collimating cylindrical microlens $2,2 \mathrm{~mm}$ in radius and optical multimode fiber 50/125 $\mu \mathrm{m}$. The end of the fiber was fixed in a metallic tube. A VHGT filters are ideal microoptical beam distribution element having high diffraction efficiency and very low insertion loses and optical crosstalk. The volume holographic transmission gratings are an extremely accurate and temperaturestable means of filtering a narrow band optical spectrum.

We measured the diffraction angles as first step, to determine geometry of location of the receiving and transmitting micromodule $\theta_{\text {diff }}=18.4^{\circ}$ for $\lambda_{1}=1490$ $\mathrm{nm}$ and $\theta_{\text {diff }}=19.1^{\circ}$ for $\lambda_{2}=1550 \mathrm{~nm}$ and determine Bragg constants of the VHGT after (1),

$$
\Lambda=\frac{\lambda_{B}}{2 \sin \left(\frac{\theta_{\text {diff }}}{2}\right)}
$$

where $\Lambda \square$ is the Bragg constant, $\lambda_{B}$ is the Bragg wavelength and $\theta_{\text {diff }}$ is the Bragg diffraction angle of a grating. The Bragg constant $\Lambda=4.66 \mu \mathrm{m}$ for $\lambda_{1}=$ $1490 \mathrm{~nm}$ and $\Lambda=4.671 \mu \mathrm{m}$ for $\lambda_{2}=1550 \mathrm{~nm}$ was calculated after (1). 
The diffraction efficiency given by (2) was calculated $\eta=99.8 \%$ for wavelength 1490 and $\eta=\square 97.4 \%$ for $1550 \mathrm{~nm}$. The results of the measuring are shown in Fig. 1,2 and Tab. 1. For measuring was used ten axis micromanipulator and Beam Profiler BP104-IR.

$$
\eta_{B}=\sin ^{2}\left(\frac{\pi \Delta n d}{\lambda_{B} \cos \alpha_{n}}\right)
$$

where $\alpha_{n}$ is the Bragg-matched incident angle in the medium, $\Delta n$ is grating strength refraction index modulation, $d$ is thickness of the grating

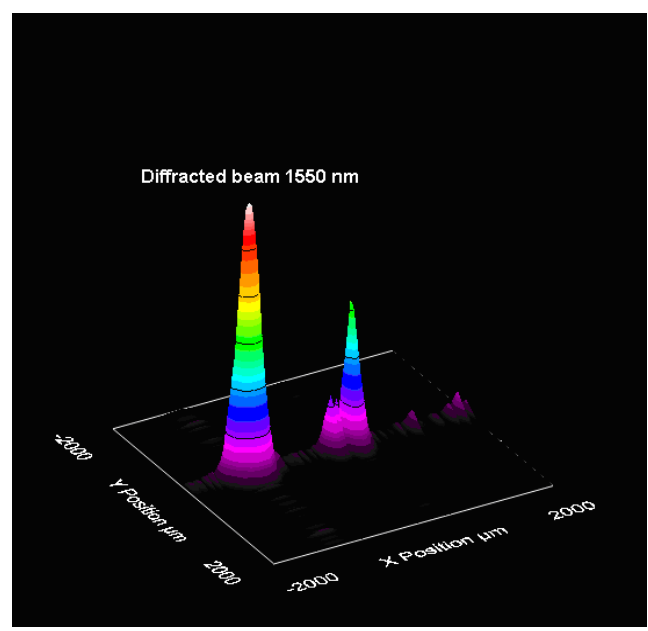

Fig. 1: The diffracted beam for the wavelength $\lambda_{1}=1550 \mathrm{~nm}$

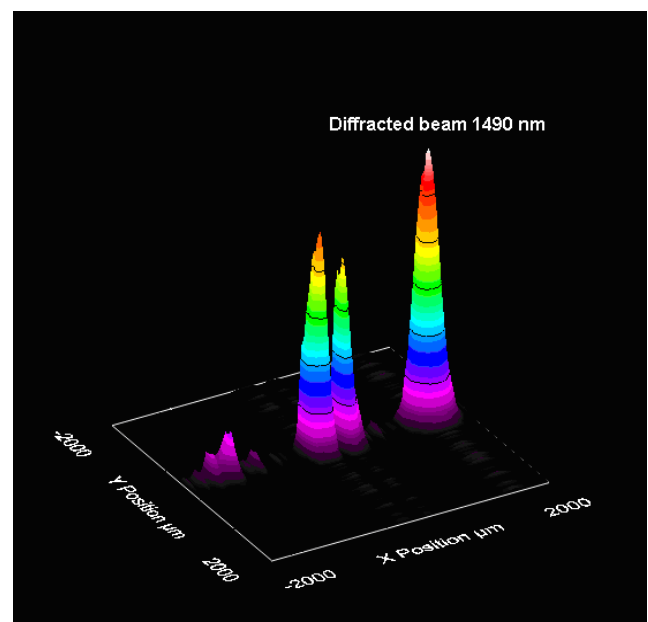

Fig. 2: The diffracted beam for the wavelength $\lambda_{2}=1490 \mathrm{~nm}$

Table 1: The diffraction efficiency of the VHGT

\begin{tabular}{cc}
\hline$\lambda_{\boldsymbol{l}}[\mathbf{n m}]$ & $\boldsymbol{\eta}_{\boldsymbol{B}}[\%]$ \\
\hline 1490 & 73.36 \\
1550 & 74.42 \\
\hline
\end{tabular}

It was founded that the measured diffract efficiency $\eta=73.6 \%$ for wavelength $1550 \mathrm{~nm}$ was different from the theoretical computed value $\eta=\square 97.4 \%$. This phenomenon comes from a grating system imper-fection and a material optical dissipation.
The minimal optical crosstalk of the optical beam for both wavelength was very important requirement to the reach good BER. The optical crosstalk was given by (3), (4)

$$
A_{\lambda}=\frac{P_{1 \lambda}}{P_{2 \lambda}}[\%]
$$

$$
a_{\lambda}=10 \log \frac{P_{1 \lambda}}{P_{2 \lambda}}[d B]
$$

where $\mathrm{P}_{\square \lambda \square}$ is optical diffracted power, $\mathrm{P}_{2 \lambda \square}$ is optical power diffracted to direction opposite wavelength. For BER $=10^{-9}$ it was needed $a_{\lambda}>11 \mathrm{~dB}$. The results of the measuring is shown in Fig. 3,4 and Tab.2

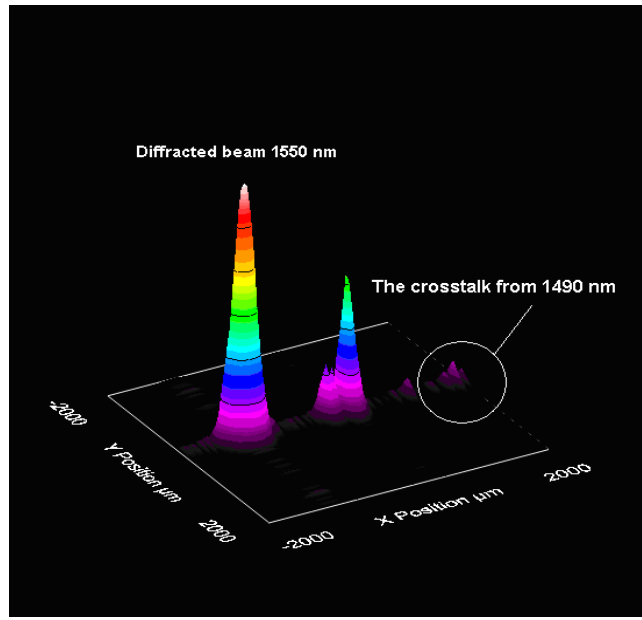

Fig. 3: The optical crosstalk for the wavelength $\lambda_{1}=1550 \mathrm{~nm}$

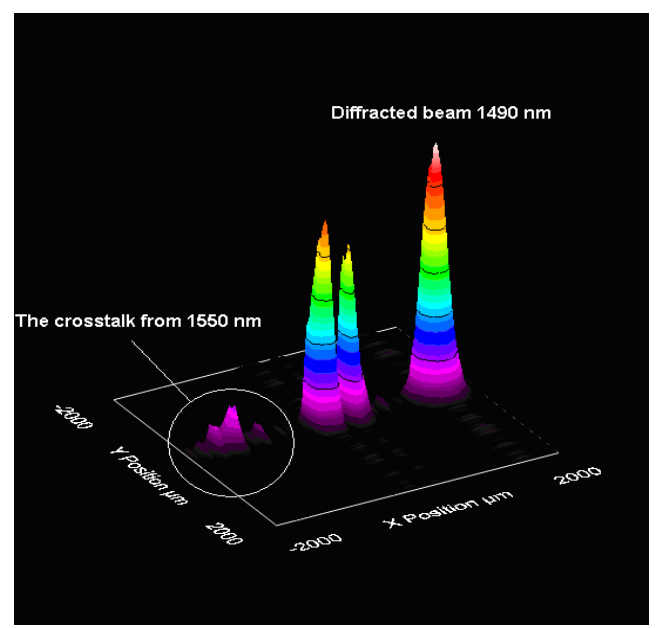

Fig. 4: The optical crosstalk for the wavelength $\lambda_{2}=1490 \mathrm{~nm}$

Table 2: The diffracted efficiency of the VHGT

\begin{tabular}{ccc}
\hline$\lambda_{\boldsymbol{l}}[\mathbf{n m}]$ & $A_{\lambda}[\%]$ & $a_{\lambda}[\boldsymbol{d} \boldsymbol{B}]$ \\
\hline 1490 & 4,95 & 13,05 \\
\hline
\end{tabular}




\section{0}

4,70

13,28

\section{The optoelectronic receiver micromodule}

Our work was concentrated on design and construction of a microwave hybrid optoelectronic receiver micromodule [5], where the PIN photodiode was connected by microstripe line to input of the HBT amplifier. The all parts are placed on the composite material substrate.

The theoretical analysis describes the microstrip connection between the PIN photodiode and the input of the HBT amplifier by the small signal equivalent circuit. For frequency response analysis we used the small signal equivalent circuit of the $\mathrm{OE}$ receiver input Fig.5

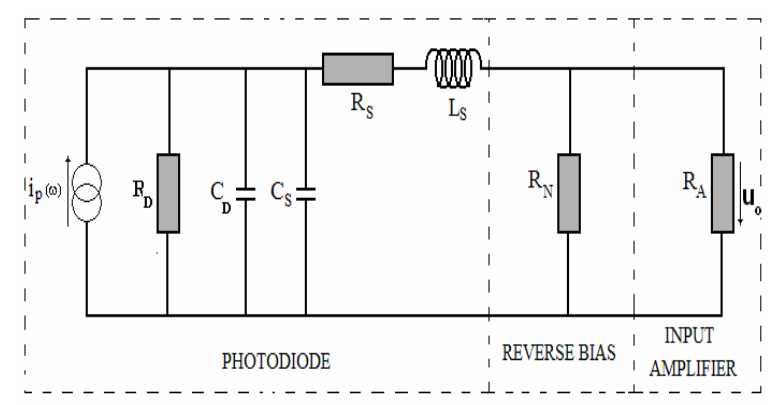

Fig. 5: The small signal equivalent circuit of the PIN photodiode with SMD assembly and input of the HBT amplifier

where

$i_{p}(\omega)$ - PIN photodiode photocurrent source

$C_{D}$ - capacitance of depletion layer $0.5 \mathrm{pF}$

$C_{S}$ - stray capacitance $1 \mathrm{pF}$

$R_{s}$ - series resistance $10 \Omega$

$R_{D}$ - dynamic resistance of the PIN photodiode $1 \mathrm{k} \Omega$

$L$ - inductance generated by the photodiode SMD carrier

$R_{N}$ - thin film resistor for reverse bias voltage $2 \mathrm{k} \Omega$

$R_{A}$ - input impedance $50 \Omega$ of the ideal amplifier

The PIN photodiode (C30606ECER from Judson Technologies) chip connection to the microstrip waveguide is composed from compensation inductance made by gold microstrip on the alumina carrier $L_{1}$ and gold wire connection $L_{2}$. For calculation frequency response limit it was counted inductance $L$ given by (5).

$$
L=L_{1}+L_{2}
$$

For the cutoff angle frequency $\omega_{\mathrm{T}}$ of the module complex transition $\mathrm{Z}_{\mathrm{T}}$ impedance characteristic (6) was derived

$$
\left|Z_{\mid}\right|=\frac{R_{2} R_{P}}{\left.\left(R_{D}+R_{S}+R_{P}-\omega L C_{D} R_{D}\right)^{2}+\omega^{2}\left(L+R_{D} R_{S} C_{D}+R_{D} R_{P} C_{D}\right)^{2}\right]^{122}}
$$

The cut off angle frequency $\omega_{\mathrm{T}}$ was derived as root of the transcendent equation (7)

$$
\left|Z_{T} \square\left(\omega_{\mathrm{T}}\right)\right|=\left|Z_{T}(0)\right| / 2^{1 / 2}
$$

where $\left|Z_{T}(0)\right|$ is module of the impedance for $\square \omega=$ $0, R_{P}$ is parallel $R_{A}$ and $R_{N}$ combination.

The limit frequency $\mathrm{f}_{\mathrm{T}}$ received by solve equation (7) was $\mathrm{f}_{\mathrm{T}}=2.78 \mathrm{GHz}$. The module $\left|Z_{T}(0)\right|=46.07$ $\Omega \square \square \square \square, \mathrm{C}_{\mathrm{T}}=1.5 \mathrm{pF}$ and $\mathrm{L}=4.5 \mathrm{nH}$. The small signal equivalent circuit presented in the Fig.5 was implemented for simulation in Win Mide program. The capacity of depletion layer $C_{D}$ was a function of reverse bias voltage and for $5 \mathrm{~V}$ is catalog value 0.50 pF. $C_{s}$ is stray capacity signal connection PIN photodiode SMD assembly. For good high frequency response it is essential to be $C_{D}$ and $C_{s}$ kept as low as possible. After that it is necessary to reduce $R_{A}$ or to provide high-frequency equalization. The inductance and capacity generated by the photodiode SMD carrier was simulated to analyze its influence on the device. The measured and simulated results at the frequency range $0.1-3.5 \mathrm{GHz}$ is shown in the Fig. 6 .

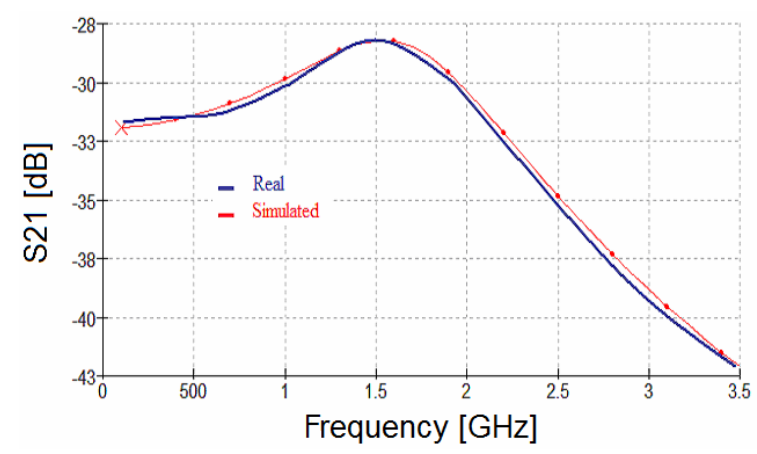

Fig. 6: The measured and simulated $S_{21}$ modulation characteristic of the OE receiver micromodule for $R_{s}=10 \Omega, L_{S}=4.8$ $\mathrm{nH}, C_{e q}=C_{D} / / C_{s}=1.5 . \mathrm{pF}$

The simulation and measurement of $S_{21}$ modulation characteristic reveal that the limit frequency $\mathrm{f}_{\mathrm{T}}$ of the $\mathrm{OE}$ receiver was $2.5 \mathrm{GHz}$. The bandwidth of the $\mathrm{OE}$ receiver is limited by the capacity depletion layer $C_{D}$ of the photodiode and $C_{S}$ stray capacity of the contact spots. The inductance distributed along the signal way between PIN photodiode and input of the HBT amplifier shift the bandwidth of the OE receiver micromodule from $1.91 \mathrm{GHz}$ to $2.5 \mathrm{GHz}$ with reasonable ripple $3 \mathrm{~dB}$.

\section{DESIGN OF MICROOPTICAL WDM TRANSCEIVER TRx}

The WDM transceiver TRx has been constructed using system of a four micromodules in the new circle topology set on the alumina or composite substrate. The fundamental layout of the hybrid 
integrated microoptical WDM transceiver is given on Fig. 7.

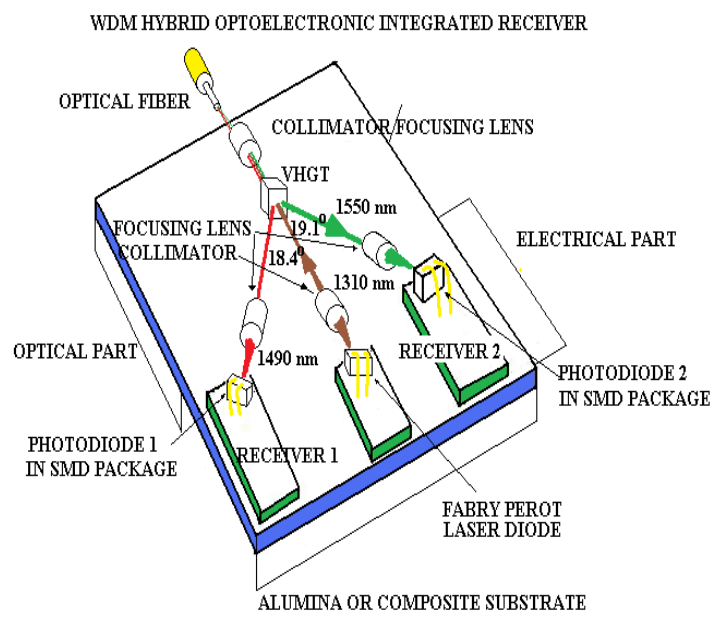

Fig. 7: WDM type triplex transceiver TRx

The triplex optical micromodule was composed of VHGT filter with collimating cylindrical microlens and optical multimode fiber. This transmission type grating filter has high diffraction efficiency, see Tab. 1 , very low insertion losses 0.14 to $0.29 \mathrm{~dB}$ and optical crosstalk after Tab.2. The optoelectronic part of TRx crate a two OE receiver micromodules with decollimation lenses. The hybrid microwave $\mathrm{OE}$ receivers uses InGaAs PIN photodiodes and HBT microwave amplifier made by thin layer hybrid integration technology. The optical beams were coupled on the PIN photodiodes active surface by decollimating lens. The optoelectronic transmitter micromodule for $\lambda_{3}=1310 \mathrm{~nm}$ upstream radiation uses Fabry-Perot InGaAsP laser diode fix on metallic submount with a microwave modulator and optical average power feed back control electronics. The feed back control was realized by ADN 2830A integrated circuit from Advance Semiconductor. The optical microisolator and collimation lens separate laser diode from nondiffracted radiation of the VHGT downstream beams.

\section{CONCLUSIONS}

In the paper we report about design and construction of a WDM bidirectional transceiver module TRx, which was design and constructed for subscribe part passive optical networks with fiber to the home topology (PON-FTTH). The TRx uses a microoptics hybrid integration technology, with volume holographic Bragg grating triplex filter (VHGT) for three wavelength MUX/DEMUX function.

Our optical WDM transceiver TRx has been constructed using system of a four micromodules in the new circle topology. The triplex optical micromodule is composed of VHGT filter with collimating cylindrical microlens and multimode optical fiber. The diffraction angles, efficiencies and insert losses of the triplex VHGT filter were verified by the beam analyzing system Beam Profiler BP 104IR from Thor Labs. The diffraction angle was 18.4 and 19.1 degrees for wavelength 1490 and $1550 \mathrm{~nm}$. The measured diffraction efficiency was $73.36 \%$ and $74.42 \%$ with insert losses below $0.3 \mathrm{~dB}$. The next two optoelectronic receiver micromodules include collimation lenses and hybrid microwave $\mathrm{OE}$ receivers. The optoelectronics receivers were made by thin layer hybrid integration technology. The optoelectronic transmitter micro-module uses FabryPerot InGaAsP laser diode with optical microisolator, microwave modulator and optical power feed back control electronics.

Alternatively we design a optical WDM transceiver TRx made by the polymer PLC (planar lightwave circuit) hybrid integration technology with a epoxy novolak polymer NANO ${ }^{\mathrm{TM}}$ SU-8 2000 monomode ridge waveguides and graded planar optical interference filters.

\section{ACKNOWLEDGMENTS}

Our research is supported by the research program MSM6840770014 of the Czech Technical University in Prague.

\section{REFERENCES}

[1] K.Kato at all., "PLC Hybrid Integration Technology and Its Application to Photonic Components," IEEE J.of Selected Topics in QE, vol. 6, p.4-13, Jan. 2000.

[2] C.H. Yeh at all, " Recent Research on Fiber Access Systems for FTTH Networks in Taiwan," IEEE LEOS Newsletter, vol. 22, pp. 15-19, Feb. 2008.

[3] C.Young-Tak, P.Yoon-Jung, at all, " Fabrication of a TFF-Attached WDM-Type Triplex Transceiver Module Using Silica Hybrid Integration Technology," J. Light. Tech., vol. 24, pp. 50315038, Dec. 2006.

[4] J. W. Goodman, Introduction to Fourier Optics, 2nd edition, McGraw-Hill, NY, USA, 1996.

[5] V.Jeřábek, J.A.Arciniega, "Hybrid Optoelectr. Receiver with Gigahertz Bandwidth," Electronic Horizon, vol. 65, pp. 22-25, No. 3, 2009. 